\title{
Status of MODIS Spatial and Spectral Characterization and Performance
}

\author{
Dan Link ${ }^{1}$, Zhipeng Wang ${ }^{1}$, and Xiaoxiong Xiong ${ }^{2}$ \\ ${ }^{1}$ Science Systems and Applications Inc., 10210 Greenbelt Rd, Lanham, MD 20706 \\ ${ }^{2}$ Sciences and Exploration Directorate, NASA/GSFC, Greenbelt, MD 20771
}

\begin{abstract}
Since launch, both Terra and Aqua MODIS instruments have continued to operate and make measurements of the earth's top of atmospheric (TOA) radiances and reflectance. MODIS collects data in 36 spectral bands covering wavelengths from 0.41 to $14.4 \mu \mathrm{m}$. These spectral bands and detectors are located on four focal plane assemblies (FPAs). MODIS on-board calibrators (OBC) include a spectro-radiometric calibration assembly (SRCA), which was designed to characterize and monitor sensor spatial and spectral performance, such as onorbit changes in the band-to-band registration (BBR), modulation transfer function (MTF), spectral band center wavelengths $(\mathrm{CW})$ and bandwidths $(\mathrm{BW})$. In this paper, we provide a status update of MODIS spatial and spectral characterization and performance, following a brief description of SRCA functions and on-orbit calibration activities. Sensor spatial and spectral performance parameters derived from SRCA measurements are introduced and discussed. Results show that on-orbit spatial performance has been very stable for both Terra and Aqua MODIS instruments. The large BBR shifts in Aqua MODIS, an issue identified pre-launch, have remained the same over its entire mission. On-orbit changes in $\mathrm{CW}$ and BW are less than $0.5 \mathrm{~nm}$ and 1 $\mathrm{nm}$, respectively, for most VIS/NIR spectral bands of both instruments.
\end{abstract}

Keywords:MODIS, calibration, spatial, spectral, SRCA, BBR, MTF, center-wavelength, bandwidth

\section{INTRODUCTION}

A large amount of the earth view data has been collected by the Moderate Resolution Imaging Spectroradiometer (MODIS) onboard the Terra and Aqua spacecraft, each operating on a sun-synchronous orbit. MODIS data is used by scientists to study the earth's land, ocean, and atmospheric conditions both for shortterm observations and long-term studies over the last 16 years. MODIS collects data over 36 spectral bands at spectral wavelengths ranging from $412 \mathrm{~nm}$ to 14.24 microns. The MODIS detectors are mounted among four focal plane assemblies (FPA) separated according to spectral range. The responses of the 20 reflective solar bands (RSB) are calibrated on-orbit using a solar diffuser (SD), which is illuminated by the sun through a SD port. A SD screen can be moved over the SD port to allow calibration of high-gain bands. A solar diffuser stability monitor (SDSM), which takes alternating views of the illuminated SD and the sun, tracks the relative change of the diffuser reflectivity at a range of wavelengths from 412 to $936 \mathrm{~nm}$. The remaining 16 bands are thermal emissive bands that are actively cooled and kept at a constant temperature. These bands are characterized by a blackbody thermal source along with regularly scheduled warm-up/cool-down (WUCD) cycles [1-7]. 
A unique on-board calibrator to the MODIS instrument is the spectro-radiometric calibration assembly (SRCA). The SRCA is designed with its own internal light sources and can provide radiometric, spatial, and spectral characterization of MODIS bands. The MODIS scan cavity and its on-board calibrators (SD, SDSM, $\mathrm{BB}$, and SRCA) are illustrated in Figure 1.

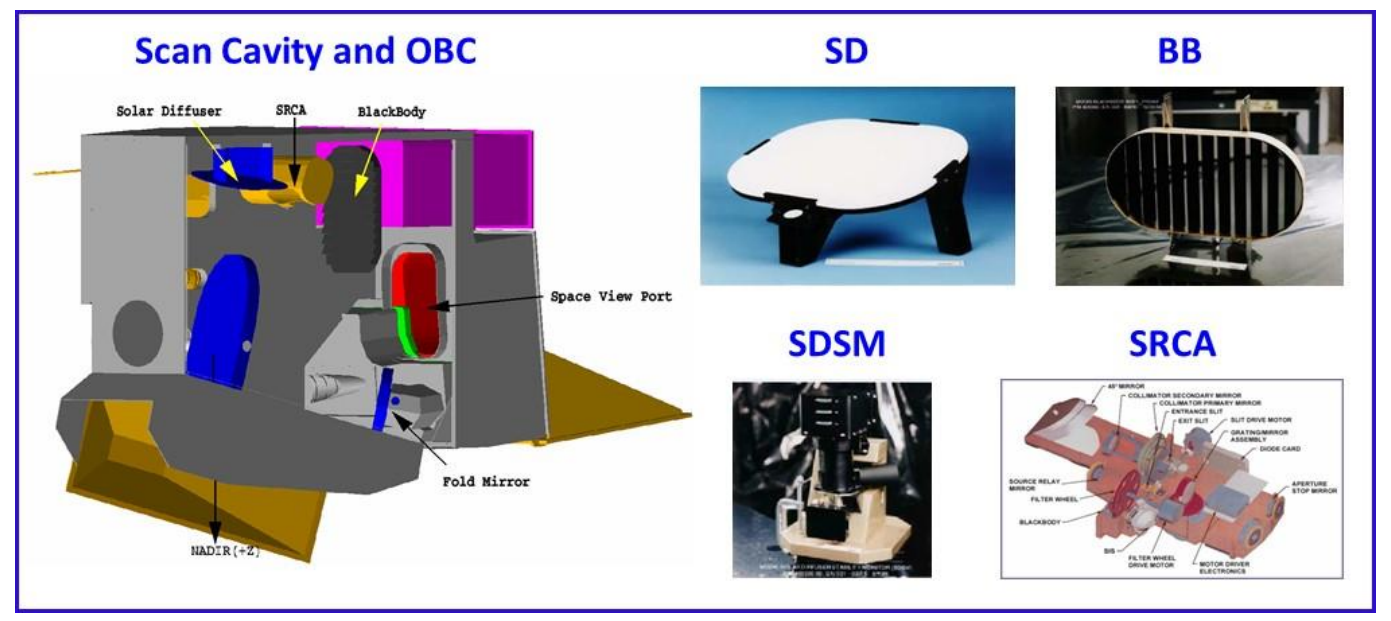

Figure 1. MODIS scan cavity and on-board calibrations (SD, SDSM, BB, and SRCA)

The objective of this paper is to review on-orbit performance of MODIS spatial and spectral properties as measured by the SRCA. The following section is a review of the calibration activities and any changes to the schedule and configurations that occurred throughout the missions. The third section provides a brief description of the methodology used to determine the characterization results. Results for both Terra and Aqua and associated discussions are presented in section 4. The paper concludes with a summary of the topics presented. As demonstrated, the SRCA continue to operate and perform its design functions. The spatial performance has been very stable for both Terra and Aqua MODIS. For VIS and NIR spectral bands, on-orbit changes in their $\mathrm{CW}$ and $\mathrm{BW}$ continue to be within their specified requirements.

\section{ON-ORBIT CALIBRATION ACTIVITIES}

Equipped with a suite of on-board calibrators, MODIS instrument calibrations are performed regularly. The frequency of on-orbit calibrations has changed during the mission. Early mission calibrations occurred frequently, daily in some instances, to characterize the system once on-orbit. Over time, the calibration schedule has reduced in frequency to preserve limited lifetime components. For example, the number of solar diffuser (SD) calibrations, which involves opening the SD door and screen, has been reduced from daily to once every three weeks, with opening the SD screen only once every six weeks. Calibration frequency can also be affected by changes to the instrument. On May 5, 2003, the Terra SD door failed to properly move as commanded. From that part onward, the SD door has been left open with the SD screen in place. This prevents Terra MODIS from performing SD calibrations with the screen open, reducing the calibration signal for the high-gain ocean bands. For nominal operation, the blackbody is set at a fixed temperature (290 K for Terra and 
$285 \mathrm{~K}$ for Aqua). In addition, the blackbody calibration is performed via a quarterly warm-up/cool-down cycle. Near-monthly roll maneuvers allow the moon to be viewed through the instrument space view (SV) port. Measurement of the moon provides an alternate source of calibration for the RSB [4-10].

The SRCA is designed to operate in three different calibration modes. When in radiometric mode, the SRCA lamps are turned on in combination to provide MODIS bands with a signal within their dynamic range. The lamps can be controlled with constant current or variable current to achieve constant radiance. Constant radiance is achieved through a feedback loop with a photodiode mounted outside of the spherical integration source (SIS), which houses the lamps. The spatial calibration mode uses the same SRCA lamps in combination along with one of two spatial reticles that shape the outgoing light for characterization in either the along-scan or the along-track directions. Using a diffraction grating, mounted on a finely controlled rotation stage, in combination with spectral band-pass filters, the SRCA can produce monochromatic signals with wavelengths from $410 \mathrm{~nm}$ to 1.0 micron on its spectral calibration mode [11-15].

Spatial calibrations yield information for all 36 MODIS bands and can be used to determine the band-to-band registration (BBR) in the along-scan and along-track directions, as well as the detector-to-detector registration (DDR) and modular transfer function (MTF) in the along-scan direction. Spatial calibrations are scheduled once for each instrument every 12 weeks (84 days). This schedule yields at least four calibrations per instrument per year. Due to limitations of the transmission of the didymium filter, used as a reference for wavelength selection, the SRCA in spectral calibration mode cannot be used to characterize bands with wavelengths greater than 1 micron. The spectral calibration can be used to characterize 16 of the 20 MODIS RSB. The current operational schedule performs spectral calibrations 16 weeks (112 days) apart, yielding at least three calibrations per instrument, per year. Spectral calibrations provide characterization of detector-level relative spectral responses (RSRs) allowing for measurement of center wavelengths and bandwidths of the subset of MODIS bands. Each calibration is designed to use lamp configurations for $30 \mathrm{~W}, 20 \mathrm{~W}, 10 \mathrm{~W}$, and $1 \mathrm{~W}$ calibrations. Additionally, a neutral density (ND) filter is available to produce intermediate signals used in the 10W/ND and $1 \mathrm{~W} / \mathrm{ND}$ cases.

Each SRCA instrument has experienced changes on-orbit leading to changes in the operational configuration and calibration activities. Each SRCA launched with four operational 10W lamps, two $1 \mathrm{~W}$ lamps, and a thermal source with one of each type of lamps designated as an on-orbit spare. In April 2003, Aqua MODIS SRCA 10W lamp \#2 was taken out of service due to reduced signal and lamp \#3 was put into service. A similar situation occurred for Terra MODIS in November 2004. Lamp \#3 on Aqua MODIS was taken out of service in June 2005. Unfortunately, with only two 10W lamps available, the Aqua MODIS SRCA could no longer perform a $30 \mathrm{~W}$ calibration that is more suitable for certain spectral bands. Some bands were able to use the signal from $20 \mathrm{~W}$ calibrations while the SNR was not high enough for band 8 (wavelength $412 \mathrm{~nm}$ ) to make valid measurements. At this point, both instruments began running in constant current mode, instead of constant radiance mode. This configuration puts less stress on the system and the lifetime of the system was deemed more important than providing radiometricly precise calibrations with no $30 \mathrm{~W}$ calibration possible. Even with this change, lamp \#3 on Terra MODIS failed to operate nominally in early 2006, leaving both MODIS instruments with only two operational 10W lamps. 


\section{METHODOLOGIES}

\section{1 spatial calibration}

Figure 2 shows the opto-mechanical system of MODIS SRCA. One of the key components is a grating/mirror assembly, which can place its mirror or the grating side in the optical configuration to perform sensor spatial or spectral characterization. In spatial mode, the SRCA is essentially an optical relay system that projects the images of the reticles placed at its exit slit onto the FPAs. When the SRCA is configured for along scan spatial characterization, a reticle with a slit equivalent to a $5 \mathrm{~km}$ (scan direction) by $12 \mathrm{~km}$ (track direction) instantaneous field of view (IFOV) is used. As MODIS scan mirror rotates, the responses from each detector as a function of data sample frame are recorded. To improve spatial characterization precision, a series of delays of data sampling time are also applied. This operation enables data samples to be collected at sub-frame intervals. A combined response profile (in digital number) is illustrated in Figure 3 (left) for Terra MODIS band 9 detector 5. From each detector's response vs sample frame, a centroid value, which is equivalent to the detector position in the scan direction, can be determined. The centroid values from two detectors or two bands (averaged over their detectors) can be used to derive their DDR or BBR.

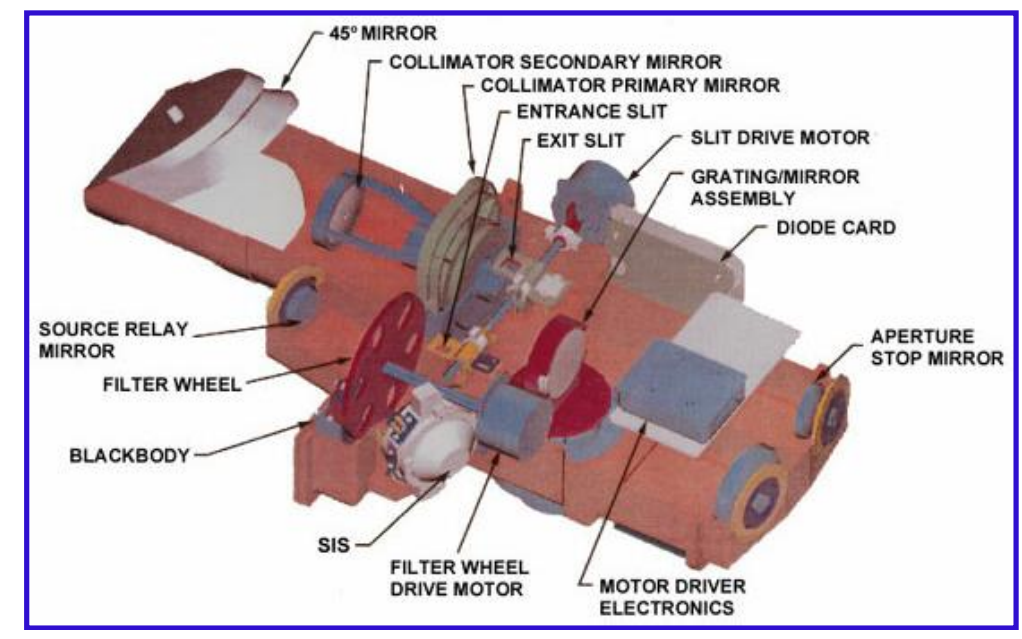

Figure 2. MODIS on-board spectro-radiometric calibration assembly (SRCA) and its key components.
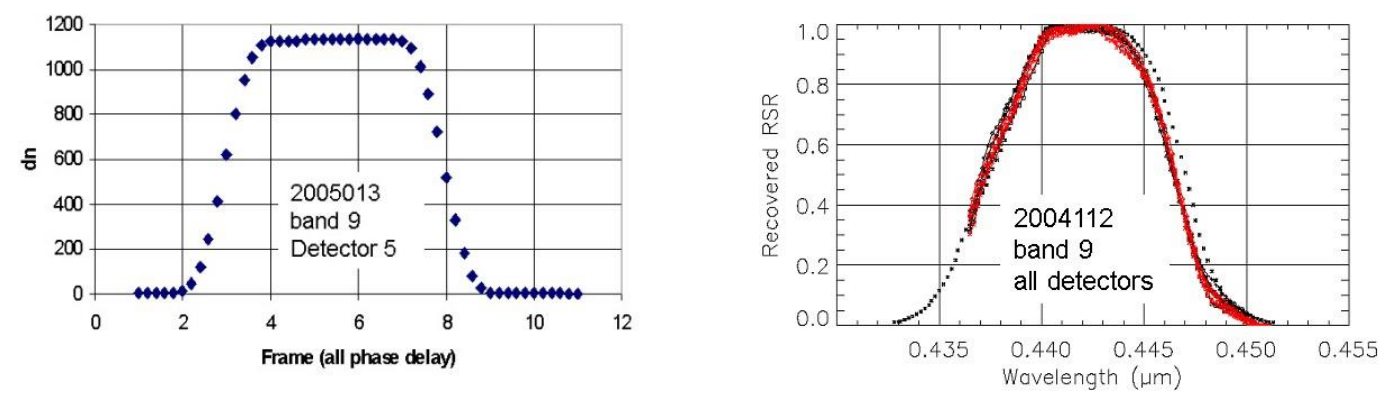

Figure 3. Left: Terra MODIS band 9 response as a function of data sample frame (detector 5, day 2005013). Right: Terra MODIS band 9 detector response as a function of wavelength (all detectors, day 2004112). 
For track direction spatial characterization, a reticle with 4 stepped openings is used. Responses of multiple detectors from the same spectral band are used to derive the BBR. No DDR is derived from this operation as some of the detectors are not illuminated. Since each detector has its own gain, a response normalization is applied among detectors within a spectral band. More details on MODIS SRCA spatial calibration methodologies for both along scan and along track direction can be found from previous publications [11-12]. In addition to BBR, the SRCA along scan spatial characterization can also be used to derive the Modulation Transfer Function (MTF).

\section{2 spectral calibration}

A grating is used when SRCA is operated in its spectral mode. In this configuration, the system consists of a monochromator and a collimator. Different grating orders are used in order to cover a wide wavelength range. More importantly, the SRCA is capable of performing internal wavelength calibration, enabling correction for any potential wavelength shifts that could be caused due to on-orbit degradation of its mechanical and/or optical components. This function is achieved through the use of a didymium filter that has extremely stable and well-calibrated spectral peaks and two silicon photo-diodes (SiPD), a calibration SiPD and a reference SiPD. A standard grating equation that relates the wavelength $(\lambda)$ and grating angle $(\theta)$ is used for spectral calibration. Apart from being used for internal wavelength calibration, the responses from the reference SiPD are also needed to remove the source spectrum in retrieving sensor relative spectral response (RSR). Figure 3 (right) shows a recovered RSR for Terra MODIS band 9 (all detectors). Also plotted is the pre-launch measured RSR (dotted back line). Due to SRCA design and operation limitations, on-orbit measurements do not cover the tail of the RSR. Nevertheless, on-orbit RSR provides sufficient information to monitor its on-orbit changes. In addition to SRCA source spectrum, the recovered RSR has corrected for the effect due to SRCA slit with finite width. From on-orbit RSR, the center wavelength and bandwidth can be calculated. More details on SRCA spectral calibration methodologies can also be found from a number of references [13-15].

\section{RESULTS AND DSICUSSION}

The results form Terra MODIS on-orbit BBR, derived from spatial calibrations in the along-scan direction are presented in Figure 4 for 2001, 2005, 2010, and 2015. The BBR presented are the average results from 3-5 calibrations per year and over all valid detectors. The specification for MODIS BBR is $\pm 200 \mathrm{~m}$ and for simplicity, we report BBR measurements relative to band 1. Not all bands met this requirement in pre-launch testing, but no new bands have shifted beyond specification since launch. The BBR has been stable on-orbit. Band 30 shows the largest change of about $50 \mathrm{~m}$, which is still much less than the specification. Similar to Figure 4, Figure 5 presents the Aqua MODIS along-scan BBR measured on-orbit for four representative years 2003, 2005, 2010, and 2015. The large BBR seen in multiple bands is due to a misalignment of the SMIR and LWIR FPAs relative to the NIR and VIS FPAs, discovered during pre-launch testing. Spatial calibrations also

provide measurements of the BBR in the along-track direction. The along-track results are similar to the alongscan results with small changes year-to-year and the largest differences are due to the misalignment of cold FPAs on Aqua MODIS. 


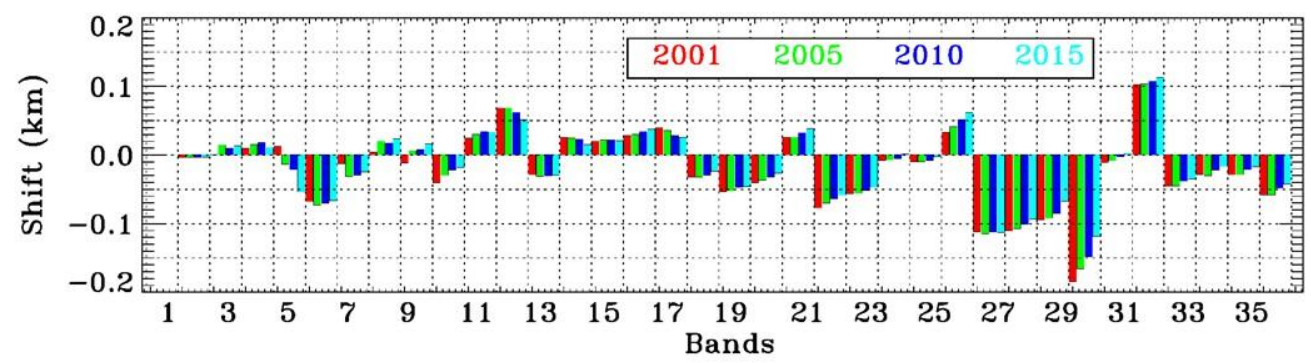

Figure 4. Terra MODIS on-orbit band-to-band registration (BBR) in along scan direction for years 2001, 2005, 2010, and 2015

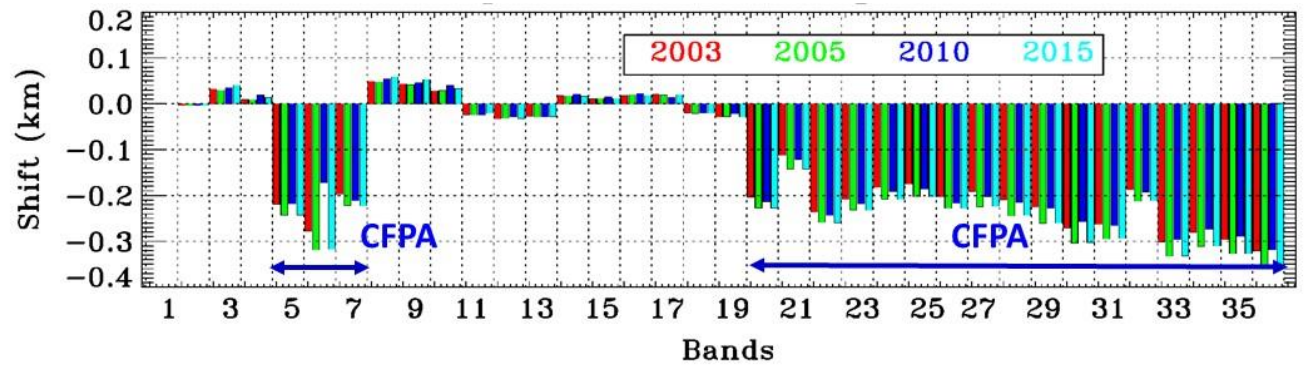

Figure 5. Aqua MODIS on-orbit band-to-band registration (BBR) in along scan direction for years 2003, 2005, 2010 , and 2015

BBR change has been shown to correlate with temperature change. The VIS and NIR FPAs are not temperature controlled, so their temperature varies seasonally with the instrument temperature. Taking a yearly average removes the impact of seasonal temperature changes in the BBR results. However, there has been a small but measurable increase in the instrument temperature throughout the MODIS missions, $3 \mathrm{~K}$ for Terra MODIS and 1.5K for Aqua MODIS, which could account for the small year-to-year trend [16]. Starting in 2007, the Aqua MODIS cold focal plane temperature has not been able to stay at a constant temperature, but after a peak in 2013, the temperature trending has begun to lower closer to the nominal set point. The change in temperature for this period could explain the BBR change seen for Aqua in 2010 and the return to 2005-like results in 2015.

The MTF generated from SRCA spatial calibration results are within specifications for most MODIS bands. The specification for MTF is defined at multiple percentages $(0.25,0.5,0.75$, and 1.0) of Nyquist frequency. Only Terra band 1, a $250 \mathrm{~m}$ resolution band, falls below the specification of 0.3 at Nyquist frequency starting in year 2013. The remaining bands and specification levels continue to be met. After more than 16 years onorbit, the slight degradation of the instrument at the highest resolution exceeds the expectation of a 6-year design life.

The calculated center wavelengths for Terra MODIS bands using SRCA spatial calibrations are presented in Figure 6 for years 2001, 2005, 2010, and 2015. Spectral results for band $2(\lambda=858 \mathrm{~nm})$ are not reported because the on-orbit configuration (specifically the grating diffraction order) is different than pre-launch measurement. The difference leaves band 2 without a pre-launch reference and can therefore only be monitored for on-orbit changes [17]. 


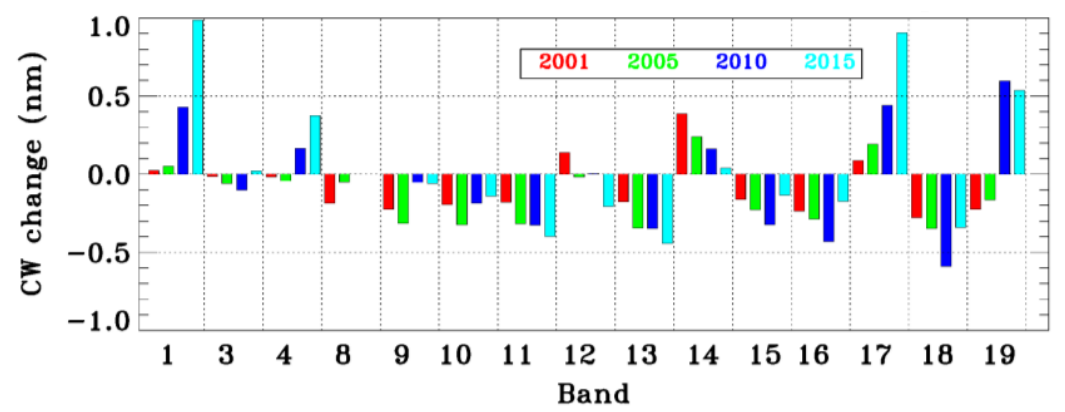

Figure 6. On-orbit changes in center wavelengths of Terra MODIS VIS/NIR spectral bands for years 2001, 2005, 2010, and 2015.

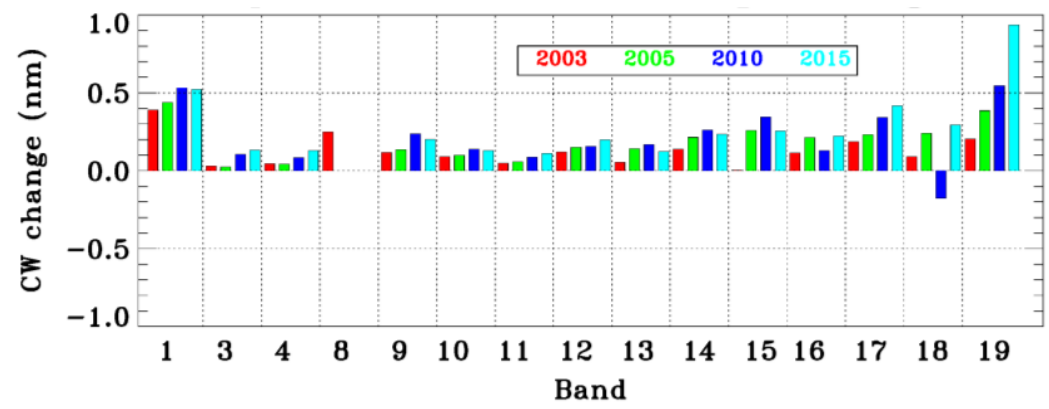

Figure 7. On-orbit changes in center wavelengths of Aqua MODIS VIS/NIR spectral bands for years 2003, 2005,2010 , and 2015

The specification for center wavelength is different for each band with the smallest tolerance of $1.0 \mathrm{~nm}$. The Terra MODIS bands shown are all within specification. Larger changes are seen in bands 1, 17, and 19, all of which have larger bandwidths and relatively large tolerances. Figure 7 presents similar information for Aqua MODIS center-wavelengths in years 2003, 2005, 2010, and 2015. The Aqua MODIS specifications are the same for Terra MODIS and all bands shown meet their center-wavelength specifications. Not shown are results for bandwidth changes for each MODIS mission. To date, all bands have bandwidth changes within specification, which are again different for each band, with bands 1 and 19 (the bands with broad bandwidth) showing relatively large change.

\section{SUMMARY}

This paper provides a brief description of MODIS SRCA spatial and spectral characterization activities and methodologies and an update of sensor spatial and spectral long-term performance. To date, Terra and Aqua MODIS have successfully operated for more than 16 and 14 years. Despite a few changes made to its operational configurations, the on-board SRCA continues to perform its design functions. Results derived from on-orbit measurements show that key spatial performance parameters have been very stable for both Terra and Aqua MODIS instruments. The large BBR shift between the bands on the cold FPA (SMIR and LWIR) and that on the warm FPA (VIS and NIR) in Aqua MODIS was an issue identified pre-launch and has remained 
unchanged over its entire mission. In general, on-orbit changes in CW and BW are less than $0.5 \mathrm{~nm}$ and $1 \mathrm{~nm}$, respectively, meeting the specified requirements.

\section{ACKNOWLEDGEMENTS}

The authors would like to acknowledge previous and current members of the MODIS Characterization Support Team (MCST) for their contributions made to operate and calibrate both Terra and Aqua MODIS instruments and especially their technical assistance and support for this work.

\section{REFERENCES}

[1] Salomonson, V., W. Barnes, X. Xiong, S. Kempler, and E. Masuoka, "An overview of the Earth Observing System MODIS instrument and associated data systems performance", Proc. IEEE International Geoscience and Remote Sensing Symposium, pp. 1174-1176, 2002.

[2] Xiong, X., V. Salomonson, and W. Barnes, "An Overview of MODIS On-orbit Calibration and Instrument Performance", Proc. IEEE International Geoscience and Remote Sensing Symposium, pp. 860-862, 2003.

[3] Xiong, X., B. Wenny, and W. Barnes, "Overview of NASA Earth Observing Systems Terra and Aqua Moderate Resolution Imaging Spectroradiometer Instrument Calibration Algorithms and On-orbit Performance", J. Appl. Remote Sens., 3(032501), 2009.

[4] Xiong X, J. Sun, W. Barnes, V. Salomonson, J. Esposito, H. Erives, and B. Guenther, "Multi-year Onorbit Calibration and Performance of Terra MODIS Reflective Solar Bands," IEEE Transactions on Geoscience and Remote Sensing, Vol. 45, No. 4, 879-889, 2007.

[5] Xiong X, K. Chiang, A. Wu, W.L. Barnes, B. Guenther, and V. Salomonson, "Multiyear On-orbit Calibration and Performance of Terra MODIS Thermal Emissive Bands," IEEE Transactions on Geoscience and Remote Sensing, 46 (6), 1790-1803, 2008.

[6] Xiong X., B. N. Wenny, A. Wu, W. Barnes, and V. Salomonson, "Aqua MODIS Thermal Emissive Bands On-orbit Calibration, Characterization, and Performance," IEEE Trans. Geosci. Remote Sens., 47(3), 803-814, 2009.

[7] Xiong, X., J. Sun, X. Xie, W. Barnes, and V. Salomonson, "On-Orbit Calibration and Performance of Aqua MODIS Reflective Solar Bands,” IEEE Trans. Geosci. Remote Sens., 48(1), 535-546, doi:10.1109/TGRS.2009.2024307, 2010.

[8] Sun J, X. Xiong, W. Barnes, and B. Guenther, "MODIS Reflective Solar Bands On-orbit Lunar Calibration," accepted for IEEE Transactions on Geoscience and Remote Sensing, Vol. 45, No. 7, 2383-2393, 2007.

[9] Xiong, X., King, M. D., Salomonson, V. V., Barnes, W. L., Wenny, B. N., Angal, A., Wu, A., Madhavan, S. and Link, D. O. (2015) Moderate Resolution Imaging Spectroradiometer on Terra and 
Aqua Missions, in Optical Payloads for Space Missions (ed S.-E. Qian), John Wiley \& Sons, Ltd, Chichester, UK. doi: 10.1002/9781118945179.ch3, November 2015.

[10] Xiong, X., A. Angal, S. Madhavan, D. Link, X. Geng, B. Wenny, A. Wu, H. Chen, and V. Salomonson, "MODIS instrument operation and calibration improvements," Proc. IEEE International Geoscience and Remote Sensing Symposium, pp. 1385 - 1388, 2014.

[11] Xiong, X., N. Che, and W. Barnes, "Terra MODIS On-Orbit Spatial Characterization and Performance", IEEE Trans. Geosci. Remote Sens., vol. 43, issue 2, pp. 355-365, 2005.

[12] Che N., X. Xiong, B. Guenther, W.L. Barnes, and V.V. Salomonson, "Five Years of Terra MODIS On-orbit Spatial Characterization", Proceedings of SPIE - Earth Observing Systems X, Vol. 5882, 58821A, doi:10.1117/12.615270, 2005.

[13] Xiong, X., N. Che, and W. Barnes, "Terra MODIS On-Orbit Spectral Characterization and Performance", IEEE Trans. Geosci. Remote Sens., vol. 44, issue 8, pp. 2198-2206, 2006.

[14] Xiong X., N. Che, B. Guenther, W.L. Barnes, and V.V. Salomonson, "Five Years of Terra MODIS On-orbit Spectral Characterization", Proceedings of SPIE - Earth Observing Systems X, Vol. 5882, 58820R, doi:10.1117/12.614090, 2005.

[15] Xiong, X., T. Choi, N. Che, Z. Wang, J. Dodd, Y. Xie and W. Barnes, "Results and lessons from a decade of Terra MODIS on-orbit spectral characterization," Proceedings of SPIE - Earth Observing Missions and Sensors: Development, Implementation, and Characterization, Vol. 7862, 78620M, doi: 10.1117/12.868930, 2010.

[16] Link, D., X. Xiong, and Z. Wang, "Assessment of MODIS on-orbit spatial performance", Proc. SPIE 9639, Sensors, Systems, and Next-Generation Satellites XIX, 963910, 2015.

[17] Choi, T., X. Xiong, Z. Wang, and D. Link, "Terra and Aqua MODIS on-orbit spectral characterization for reflective solar bands ", Proc. SPIE 8724: Ocean Sensing and Monitoring V 87240Y, 2013. 